\title{
ASYMPTOTIC COMPLETION OF A MODULE WITH RESPECT TO A FILTRATION
}

\section{ERIC DAGO AKEKE and PHILIPPE AYEGNON}

UFHB de Cocody, UFRMI

22 BP 582 Abidjan 22

Côte d'Ivoire

e-mail: ericdago@yahoo.fr

E. N. S. d'Abidjan 08

BP 10 Abidjan 08

Côte d'Ivoire

\begin{abstract}
The concept of $I$-cloture of a module with respect to an ideal $I$ has been defined by Naudé and Naudé in [5]. For any filtration $f$ on a Noetherian ring $A$ and for a submodule $N$ of a finitely generated $A$-module $M$, we define the $f$-cloture $N_{\sigma_{f}}$. Some results in [5] are extended to filtrations on a ring. We show that for an AP-filtration $f$, the map $N \mapsto N_{\sigma_{f}}$ defined on a set of $f$-normal submodule of $M$ is a semiprime operation.
\end{abstract}

\section{Introduction}

Throughout this paper, the letter $A$ will denote a commutative ring with identity. A filtration on a ring $A$ is a sequence $f=\left(I_{n}\right)_{n \in \mathbb{N}}$ of ideals such that $I_{0}=A, I_{n+1} \subseteq I_{n}$ and $I_{n} I_{m} \subseteq I_{n+m}$ for all $n, m \in \mathbb{N}$. If $f=\left(I_{n}\right)$ is a filtration on the ring $A$, then $f^{(k)}=\left(I_{n k}\right)_{n \in \mathbb{N}}$ and 2010 Mathematics Subject Classification: 13A15, 13A18.

Keywords and phrases: filtration, module, ring.

Received July 28, 2016

(ㄷ) 2016 Scientific Advances Publishers 
$f^{k}=\left(I_{n}^{k}\right)_{n \in \mathbb{N}}$ are filtrations on the ring $A$ for all $k \in \mathbb{N}^{*}$. The product of two filtrations $f=\left(I_{n}\right)_{n \in \mathbb{N}}$ and $g=\left(J_{n}\right)_{n \in \mathbb{N}}$ on the ring $A$ is the filtration $f g=\left(I_{n} J_{n}\right)_{n \in \mathbb{N}}$. The set $F(A)$ of all filtrations on a ring $A$ is ordered by the relation $f=\left(I_{n}\right) \leq g=\left(J_{n}\right)$ if $I_{n} \subseteq J_{n}$ for all $n \geq 0$. A filtration $f=\left(I_{n}\right)$ on the ring $A$ is said to be approximatable by powers and is called an AP-filtration in case there exists a sequence of positive integers numbers $\left(k_{n}\right)_{n \in \mathbb{N}}$ such that $I_{k_{n} m} \subseteq I_{n}^{m}$ for all $n, m$ and $\lim _{n \rightarrow+\infty} \frac{k_{n}}{n}=1$. A filtration of submodules on an $A$-module $M$ is a sequence $\left(M_{n}\right)_{n \in \mathbb{N}}$ of submodules of $M$ such that $M_{0}=M$ and $M_{n+1} \subseteq M_{n}$ for all $n \in \mathbb{N}$.

Let $f=\left(I_{n}\right)$ be a filtration on a ring $A, M$ be an $A$-module, and $N$ be a sub-module of $M$. Let $\Lambda$ be a commutative subring of $\operatorname{End}_{A}(M)$, the ring of $A$-endomorphisms of $M$. We study the numbers $\bar{v}_{f, N}(\alpha)=\lim _{n \rightarrow+\infty}$ $\frac{v_{f, N}\left(\alpha^{n}\right)}{n}$ with $v_{f, N}\left(\alpha^{n}\right)=\sup \left\{k \in \mathbb{N}^{*} / \alpha^{n}(M) \subseteq I_{k} N\right\}$. These numbers have been introduced and studied by Naudé and Naudé in [5], in the case of $I$-adic filtration on a ring.

In Section 3, we gives some properties of the numbers $\bar{v}_{f, N}(\alpha)$. We show that if $f=\left(I_{n}\right)$ is an AP-filtration on the ring $A$, then $\bar{v}_{f, N}(\alpha)=$ $\bar{v}_{f, I_{k} N}(\alpha)$ for all $k \in \mathbb{N}^{*}$ and $\alpha \in \Lambda$ (cf. Proposition 3.1 (iii)). We obtain the following main results:

Theorem 3.1. Let $f=\left(I_{n}\right), g=\left(J_{n}\right)$ be filtrations on the ring $A$ and $N$ be a sub-module of $M$ such that $\alpha(N) \subseteq N$ for all $\alpha \in \Lambda$. Then

(a) $\frac{1}{\bar{v}_{f g, N}(\alpha)} \leq \frac{1}{\bar{v}_{f, N}(\alpha)}+\frac{1}{\bar{v}_{g, N}(\alpha)}$.

(b) $\bar{v}_{f^{n}, N}(\alpha)=\frac{1}{n} \bar{v}_{f, N}(\alpha)=\bar{v}_{f}^{(n), N}(\alpha)$ for all $n \in \mathbb{N}^{*}$ and $\alpha \in \Lambda$. 
Proposition 3.2. Let $f=\left(I_{n}\right), g=\left(J_{n}\right)$ be filtrations on the ring $A$ and $N$ be a sub-module of $M$ such that $\alpha(N) \subseteq N$ for all $\alpha \in \Lambda$.

(a) If $\bar{v}_{g, N}(\alpha) \neq 0$, then $\lim _{n \rightarrow+\infty} n \bar{v}_{f^{n}{ }_{g, N}}(\alpha)$ exists in $\overline{\mathbb{R}}$ and we have

$$
\lim _{n \rightarrow+\infty} n \bar{v}_{f^{n} g, N}(\alpha)=\bar{v}_{f, N}(\alpha) .
$$

(b) If $\bar{v}_{g, N}(\alpha)=0$, then $\lim _{n \rightarrow+\infty} n \bar{v}_{f^{n} g, N}(\alpha)=0$.

(c) If $\sqrt{f N} \subseteq \sqrt{g N}$, then $\lim _{n \rightarrow+\infty} n \bar{v}_{f^{n} g, N}(\alpha)=\bar{v}_{f, N}(\alpha)$.

In Section 4, we obtain the following results:

Theorem 4.1. Suppose that $A$ is a Noetherian ring and $M$ is finitely generated. If $N$ is $f^{n}$-normal, then $\bar{v}_{I_{n}, N}=\bar{v}_{I_{n}, \tilde{N}_{n}}$ with $\tilde{N}_{n}=$ $\sum_{\bar{v}_{f, N}(\alpha) \geq n} \alpha(M)$.

Theorem 4.2. Let $A$ be a Noetherian ring and $f=\left(I_{n}\right)_{n \in \mathbb{N}}, N, M$, and $\Lambda$ be as above. Suppose that $M$ is finitely generated and $N$ is f-normal. Then

$$
\bar{v}_{f, N}=\bar{v}_{f, N_{\sigma f}},
$$

with $N_{\sigma_{f}}=\sum_{\bar{v}_{f, N}(\alpha) \geq 1} \alpha(M)$.

Proposition 4.1. Let $A$ be a Noetherian ring and $f=\left(I_{n}\right)_{n \in \mathbb{N}}$ an AP-filtration on $A$. Assume that $M$ is a finitely generated A-module. If $N$ and $L$ are $\Lambda$-invariant and $f$-normal submodules of $M$, then we have

(i) if $N \subseteq L$, then $N_{\sigma_{f}} \subseteq L_{\sigma_{f}}$;

(ii) $\left(N_{\sigma_{f}}\right)_{\sigma_{f}}=N_{\sigma_{f}}$;

(iii) $I_{n} N_{\sigma_{f}} \subseteq\left(I_{n} N\right)_{\sigma_{f}}$ for all $n \in \mathbb{N}^{*}$. 
Theorem 3.1 is a generalization of Proposition 2.5 in [5]. Proposition 3.2 is a generalization of Proposition 2.6 in [5]. Theorem 4.1 and Theorem 4.2 are generalization of Proposition 3.2 in [5] and Proposition 4.1 is a generalization of Proposition 3.8 in loc. cit.

\section{Pseudo-Valuation on a Subring of $\operatorname{End}_{A}(M)$}

Definition 2.1. Let $R$ be a ring. A pseudo-valuation on $R$ is a map $v: R \rightarrow \overline{\mathbb{N}}=\mathbb{N} \cup\{+\infty\}$ such that:

(i) $v(0)=+\infty, v(1) \geq 0$;

(ii) $v(x+y) \geq \min (v(x), v(y))$ for all $x, y \in R$;

(iii) $v(x y) \geq v(x)+v(y)$ for all $x, y \in R$.

A pseudo-valuation $v$ on $R$ is said to be homogeneous if for all $k \in \mathbb{N}^{*}$ and for all $x \in R$ we have $v\left(x^{k}\right)=k v(x)$.

Let $f=\left(I_{n}\right)$ be a filtration on a ring $A, M$ be an $A$-module, and $N$ be a sub-module of $M$. Let $\Lambda$ be a commutative subring of $\operatorname{End}_{A}(M)$, the ring of $A$-endomorphisms of $M$. For all $\alpha \in \Lambda$, we put

$$
v_{f, N}(\alpha)=\left\{\begin{aligned}
0, & \text { if } & \alpha(M) \nsubseteq I_{1} N, \\
+\infty, & \text { if } & \forall n \in \mathbb{N}, \quad \alpha(M) \subset I_{n} N, \\
k, & \text { if } & \alpha(M) \subseteq I_{k} N \text { and } \alpha(M) \nsubseteq I_{k+1} N .
\end{aligned}\right.
$$

Proposition 2.1. Let $f, N, M$, and $\Lambda$ be as above such that $\alpha(N) \subset N$ for all $\alpha \in \Lambda$. Then the map $v_{f, N}: \Lambda \rightarrow \mathbb{N} \cup\{+\infty\}$ is a pseudo-valuation on the ring $\Lambda$. 
Proof. (i) $v_{f, N}(0)=+\infty$ and $v_{f, N}\left(i d_{M}\right) \geq 0$ follow directly from the definition of $v_{f, N}$.

Let $v_{f, N}(\alpha)=k$ and $v_{f, N}(\beta)=p$ with $\alpha, \beta \in \Lambda, k, p \in \mathbb{N}$.

(ii) If $k=p=0$, then

$$
v_{f, N}(\alpha \beta) \geq v_{f, N}(\alpha)+v_{f, N}(\beta) .
$$

Suppose that $p>0$ and $k \geq 0$. We have

$$
\alpha \beta(M) \subset \alpha\left(I_{p} N\right) \subset I_{k} I_{p} N \subset I_{k+p} N .
$$

It follows that $v_{f, N}(\alpha \beta) \geq p+k$ and we have $(*)$.

It is obvious that if $p=+\infty$, then $\alpha \beta(M) \subseteq \alpha\left(I_{n} N\right) \subseteq I_{n} N$ for all $n \in \mathbb{N}$. Therefore, $v_{f, N}(\alpha \beta) \geq n, \forall n \in \mathbb{N}$. It follows that $v_{f, N}(\alpha \beta)=+\infty$ and we have $(*)$.

(iii) Now let's show that

$$
v_{f, N}(\alpha+\beta) \geq \min \left(v_{f, N}(\alpha), v_{f, N}(\beta)\right) .
$$

It's obvious that if $k=0$ or $p=0$, then we have $(* *)$. Suppose that $k, p \in \mathbb{N}^{*}$. Then

$$
(\alpha+\beta)(M) \subseteq \alpha(M)+\beta(M) \subseteq I_{k} N+I_{p} N \subseteq I_{m} N
$$

with $m=\min (k, p)$. Therefore $v_{f, N}(\alpha+\beta) \geq m$ and we have $(* *)$.

If $k=+\infty$ or $p=+\infty$, then there exists $n_{0} \in \mathbb{N}^{*}$ such that

$$
\forall n \geq n_{0}, \quad(\alpha+\beta)(M) \subseteq I_{n} N
$$

It follows that $v_{f, N}(\alpha+\beta) \geq n$ for all $n \geq n_{0}$, hence $v_{f, N}(\alpha+\beta)=+\infty$ and we have $(* *)$. 
Proposition 2.2. Let $f=\left(I_{n}\right)_{n \in \mathbb{N}}, N, M$, and $\Lambda$ be as above such that $\alpha(N) \subset N$ for all $\alpha \in \Lambda$. Then $\bar{v}_{f, N}(\alpha)=\lim _{n \rightarrow+\infty} \frac{v_{f, N}\left(\alpha^{n}\right)}{n}$ exists in $\overline{\mathbb{R}}_{+}=\mathbb{R}_{+} \cup\{+\infty\}$.

Proof. Lemma 2.11 in [6] or Lemma 3.1 in [8].

Remark 2.1. Let $\alpha \in \Lambda$ and $k \in \mathbb{N}^{*}$. We put $w_{n}=\frac{v_{f, N}\left(\alpha^{k^{n}}\right)}{k^{n}}$ for all $n \in \mathbb{N}^{*}$. It is obvious that $w_{n}=+\infty \Rightarrow w_{n+1}=+\infty$. If $w_{n} \in \mathbb{N}^{*}$, then

$$
\frac{w_{n+1}}{w_{n}}=\frac{v_{f, N}\left(\left(\alpha^{k^{n}}\right)^{k}\right)}{k v_{f, N}\left(\alpha^{k^{n}}\right)} \geq 1,
$$

since $v_{f, N}\left(\left(\alpha^{k^{n}}\right)^{k}\right) \geq k v_{f, N}\left(\alpha^{k^{n}}\right)$. Therefore $w_{n} \leq w_{n+1}$. It follows that $\left(w_{n}\right)_{n \in \mathbb{N}^{*}}$ in an increasing sequence in $\overline{\mathbb{R}}_{+}$and we have $\bar{v}_{f, N}(\alpha)=$ $\sup \frac{v_{f, N}\left(\alpha^{n}\right)}{n}$.

Proposition 2.3. Let $f=\left(I_{n}\right)_{n \in \mathbb{N}}, N, M$, and $\Lambda$ be as above such that $\alpha(N) \subset N$ for all $\alpha \in \Lambda$. Then the map $\bar{v}_{f, N}: \Lambda \rightarrow \overline{\mathbb{R}}_{+}$is a homogeneous pseudo-valuation on the ring $\Lambda$.

Proof. (i) We easily have $\bar{v}_{f, N}(0)=+\infty$ and $\bar{v}_{f, N}\left(i d_{M}\right) \geq 0$. Let $\alpha, \beta \in \Lambda$.

(ii) For all $k \in \mathbb{N}$, we have $(\alpha \beta)^{k}=\alpha^{k} \beta^{k}$, therefore,

$$
v_{f, N}\left((\alpha \beta)^{k}\right) \geq v_{f, N}\left(\alpha^{k}\right)+v_{f, N}\left(\beta^{k}\right) .
$$

It follows that $\bar{v}_{f, N}(\alpha \beta) \geq \bar{v}_{f, N}(\alpha)+\bar{v}_{f, N}(\beta)$. 
(iii) Let us put $\lambda=\min \left(\bar{v}_{f, N}(\alpha), \bar{v}_{f, N}(\beta)\right)$. We can assume $\lambda>0$. Let $\lambda>b>0$. There exists an integer $m>0$ such that,

$$
v_{f, N}\left(\alpha^{n}\right) \geq n b \text { and } v_{f, N}\left(\beta^{n}\right) \geq n b,
$$

for all $n \geq m$. We have $(\alpha+\beta)^{n}=\sum_{p=0}^{n} \mathcal{C}_{n}^{p} \alpha^{p} \beta^{n-p}$ since $\alpha \beta=\beta \alpha$. It follows that

$$
v_{f, N}\left((\alpha+\beta)^{n}\right) \geq \min _{0 \leq p \leq n} v_{f, N}\left(\mathcal{C}_{n}^{p} \alpha^{p} \beta^{n-p}\right) \geq \min _{0 \leq p \leq n}\left(v_{f, N}\left(\alpha^{p}\right)+v_{f, N}\left(\beta^{n-p}\right)\right),
$$

Let $n \in \mathbb{N}$ such that $n \geq 2 m$.

For $p<m$, we have $n-p>m$, therefore $v_{f, N}\left(\beta^{n-p}\right) \geq(n-p) b$. It follows that

$$
v_{f, N}\left(\alpha^{p}\right)+v_{f, N}\left(\beta^{n-p}\right) \geq(n-m) b
$$

since $n-p>n-m$.

If $p \geq m$ and $p \leq n-m$, then $v_{f, N}\left(\alpha^{p}\right) \geq p b$ and $v_{f, N}\left(\beta^{n-p}\right) \geq(n-p) b$, therefore

$$
v_{f, N}\left(\alpha^{p}\right)+v_{f, N}\left(\beta^{n-p}\right) \geq p b+(n-p) b=n b \geq(n-m) b .
$$

If $p>n-m$, then $p>m$ and $v_{f, N}\left(\alpha^{p}\right) \geq p b>(n-m) b$, therefore,

$$
v_{f, N}\left(\alpha^{p}\right)+v_{f, N}\left(\beta^{n-p}\right)>(n-m) b .
$$

We obtain

$$
v_{f, N}\left(\alpha^{p}\right)+v_{f, N}\left(\beta^{n-p}\right) \geq(n-m) b,
$$

for all $n \geq 2 m$. By inequalities $(*)$ and $(* *)$, we have

$$
\frac{v_{f, N}\left((\alpha+\beta)^{n}\right)}{n} \geq \frac{n-m}{n} b
$$


for all $n \geq 2 m$. Taking the limit as $n \rightarrow+\infty$ we get

$$
\bar{v}_{f, N}(\alpha+\beta) \geq b \text {. }
$$

Taking the limit as $b \rightarrow \lambda$ we get

$$
\bar{v}_{f, N}(\alpha+\beta) \geq \lambda
$$

therefore,

$$
\bar{v}_{f, N}(\alpha+\beta) \geq \min \left(\bar{v}_{f, N}(\alpha), \bar{v}_{f, N}(\beta)\right) .
$$

(iv) Let $k \in \mathbb{N}^{*}$. We have

$$
\bar{v}_{f, N}\left(\alpha^{k}\right)=\lim _{n \rightarrow+\infty} \frac{v_{f, N}\left(\alpha^{k n}\right)}{n}=\lim _{n \rightarrow+\infty} k \frac{v_{f, N}\left(\alpha^{k n}\right)}{k n}=k \bar{v}_{f, N}(\alpha) .
$$

Therefore $\bar{v}_{f, N}$ is homogeneous.

Notation 2.1. Let $I$ be an ideal of the ring $A$ and $f_{I}=\left(I^{n}\right)_{n \in \mathbb{N}}$ be the $I$-adic filtration on $A$. Let $\alpha \in \Lambda$. We put $v_{I, N}(\alpha)=v_{f_{I}, N}(\alpha)$.

\section{Some Properties of $\bar{v}_{f, N}$}

Let $f=\left(I_{n}\right)_{n \in \mathbb{N}}, M$ and $\Lambda$ be as above. Let's give in this section some properties of $\bar{v}_{f, N}$.

Proposition 3.1. Let $L$ and $N$ be sub-modules of $M$ such that $\alpha(L) \subseteq L$ and $\alpha(N) \subseteq N$ for all $\alpha \in \Lambda$ and $g=\left(J_{n}\right)_{n \in \mathbb{N}}$ a filtration on the ring $A$.

(i) If $N \subseteq L$, then $\bar{v}_{f, N}(\alpha) \leq \bar{v}_{f, L}(\alpha)$ for all $\alpha \in \Lambda$.

(ii) If $f \leq g$, then $\bar{v}_{f, N}(\alpha) \leq \bar{v}_{g, N}(\alpha)$ for all $\alpha \in \Lambda$.

(iii) If $f=\left(I_{n}\right)$ is an AP-filtration on the ring $A$, then

$$
\bar{v}_{f, N}(\alpha)=\bar{v}_{f, I_{k} N}(\alpha)
$$

for all $k \in \mathbb{N}$ and $\alpha \in \Lambda$. 
Proof. (i) It is obvious if $\bar{v}_{f, N}(\alpha)=0$. Suppose that $v_{f, N}\left(\alpha^{k}\right)=+\infty$ with $k \in \mathbb{N}$. We have $\alpha^{k}(M) \subseteq I_{n} N \subseteq I_{n} L$ for all $n \in \mathbb{N}$, therefore $v_{f, L}\left(\alpha^{k}\right) \geq n$ for all $n \in \mathbb{N}$, it follows that

$$
\bar{v}_{f, N}(\alpha)=\bar{v}_{f, L}(\alpha)=+\infty .
$$

Let's now assume that $v_{f, N}\left(\alpha^{k}\right) \in \mathbb{N}$, for all $k \in \mathbb{N}$. For any integer $n$ such that $\alpha^{k}(M) \subseteq I_{n} N$, we have $\alpha^{k}(M) \subseteq I_{n} L$, therefore $v_{f, L}\left(\alpha^{k}\right) \geq$ $v_{f, N}\left(\alpha^{k}\right)$. It follows that

$$
\lim _{k \rightarrow+\infty} \frac{v_{f, L}\left(\alpha^{k}\right)}{k} \geq \lim _{k \rightarrow+\infty} \frac{v_{f, N}\left(\alpha^{k}\right)}{k},
$$

hence $\bar{v}_{f, L}(\alpha) \geq \bar{v}_{f, N}(\alpha)$.

(ii) Follows from the relevant definition.

(iii) Suppose that $f=\left(I_{n}\right)_{n \in \mathbb{N}}$ is an AP-filtration. Let $k \in \mathbb{N}$. By (i) we have

$$
\bar{v}_{f, I_{k} N}(\alpha) \leq \bar{v}_{f, N}(\alpha)
$$

Let $\lambda=\bar{v}_{f, N}(\alpha)$ and $\frac{p}{q} \in \mathbb{Q}$ such that $0<\frac{p}{q}<\lambda$. There exists an integer $n_{0}$ such that $\frac{v_{f, N}\left(\alpha^{n}\right)}{n} \geq \frac{p}{q}$ for all $n \geq n_{0}$. It follows that $v_{f, N}\left(\alpha^{q n}\right) \geq p n$ for all $n \geq n_{0}$. Let $\left(k_{n}\right)_{n \in \mathbb{N}}$ be a sequence of integers such that $\lim _{n \rightarrow+\infty} \frac{k_{n}}{n}=1$ and $I_{k_{n} m} \subseteq I_{n}^{m}, \forall n, m \in \mathbb{N}$. We have

$$
\alpha^{k_{n} m q}(M) \subseteq I_{k_{n} m p} N \subseteq I_{n}^{m p} N=I_{n}^{m p-1} I_{n} N \subseteq I_{n(m p-1)} I_{n} N
$$

for all $n \geq 0$ and $m \geq n_{0}$. Therefore,

$$
v_{f, I_{n} N}\left(\alpha^{k_{n} m q}\right) \geq n(m p-1) .
$$


It follows that

$$
\frac{v_{f, I_{n} N}\left(\alpha^{k_{n} m q}\right)}{k_{n} m q} \geq \frac{n p}{k_{n} q}-\frac{n}{k_{n} m q} .
$$

Taking the limit as $m \rightarrow+\infty$, we get $\bar{v}_{f, I_{n} N}(\alpha) \geq \frac{n p}{k_{n} q}$ for all $n$. Therefore,

$$
\bar{v}_{f, I_{k} N}(\alpha) \geq \lim _{n \rightarrow+\infty} \bar{v}_{f, I_{n} N}(\alpha) \geq \frac{p}{q},
$$

so that, $\bar{v}_{f, I_{k} N}(\alpha) \geq \frac{p}{q}$. Taking the limit as $\frac{p}{q} \rightarrow \lambda$, we obtain

$$
\bar{v}_{f, I_{k} N}(\alpha) \geq \bar{v}_{f, N}(\alpha) \text {. }
$$

By (1) and (2), we have $\bar{v}_{f, I_{k} N}(\alpha)=\bar{v}_{f, N}(\alpha)$.

Definition 3.1 ([5]). Let $N$ be a sub-module of $M$ and $\Lambda$ be a commutative subring of $\operatorname{End}_{A}(M)$. We define the radical of $N$ in $\Lambda$ by

$$
\operatorname{Rad}\left(N:_{\Lambda} M\right)=\left\{\alpha \in \Lambda / \exists n \in \mathbb{N}^{*}, \alpha^{n}(M) \subset N\right\} .
$$

Remark 3.1. (1) $\operatorname{Rad}\left(N:_{\Lambda} M\right)$ is an ideal of $\Lambda$.

(2) Let $I$ and $J$ be ideals of $A$. We have

$$
\operatorname{Rad}\left(\operatorname{IJN}:_{\Lambda} M\right)=\operatorname{Rad}\left(I N:_{\Lambda} M\right) \cap \operatorname{Rad}\left(J N:_{\Lambda} M\right) .
$$

(3) If $f=\left(I_{n}\right)_{n \in \mathbb{N}}$ is a filtration on $A$, then

$$
\operatorname{Rad}\left(I_{1} N:_{\Lambda} M\right)=\operatorname{Rad}\left(I_{n} N:_{\Lambda} M\right),
$$

for all $n \in \mathbb{N}^{*}$. This common value is denoted by $\sqrt{f N}$.

Theorem 3.1. Let $f=\left(I_{n}\right), g=\left(J_{n}\right)$ be filtrations on the ring $A$ and $N$ be a sub-module of $M$ such that $\alpha(N) \subseteq N$ for all $\alpha \in \Lambda$. Then
(a) $\frac{1}{\bar{v}_{f g, N}(\alpha)} \leq \frac{1}{\bar{v}_{f, N}(\alpha)}+\frac{1}{\bar{v}_{g, N}(\alpha)}$.
(b) $\bar{v}_{f^{n}, N}(\alpha)=\frac{1}{n} \bar{v}_{f, N}(\alpha)=\bar{v}_{f^{(n)}, N}(\alpha)$ for all $n \in \mathbb{N}^{*}$ and $\alpha \in \Lambda$. 
Proof. (i) If $\bar{v}_{f, N}(\alpha)=0$ or $\bar{v}_{g, N}(\alpha)=0$, then we have inequality (a).

Let's now assume that $\bar{v}_{f, N}(\alpha)=\gamma>0$ and $\bar{v}_{g, N}(\alpha)=\delta>0$. Let $\frac{p}{q}, \frac{n}{m} \in \mathbb{Q}$ such that $0<\frac{p}{q}<\gamma$ and $0<\frac{n}{m}<\delta$. There exists $k, l \in \mathbb{N}^{*}$ such that

$$
\alpha^{q k}(M) \subseteq I_{p k} N \quad \text { and } \quad \alpha^{n l}(M) \subseteq J_{m l} N
$$

It follows that

$$
\left(\alpha^{q k}\right)^{m l}(M) \subseteq I_{p k}^{m l} N \subseteq I_{p k l m} N \text { and }\left(\alpha^{n l}\right)^{p k}(M) \subseteq J_{m l}^{p k} N \subseteq J_{m l p k} N,
$$

hence,

$$
\begin{aligned}
\alpha^{(q m+n p) k l}(M) \subseteq \alpha^{q m k l}\left(\alpha^{n p k l}(M)\right) & \subseteq \alpha^{q m k l}\left(J_{m l p k} N\right) \\
& \subseteq J_{m l p k} \alpha^{q m k l}(N) \subseteq I_{p k l m} J_{m l p k} N .
\end{aligned}
$$

We obtain $\alpha^{(q m+n p) k l}(M) \subseteq I_{m l p k} J_{m l p k} N$, so that

$$
v_{f g, N}\left(\alpha^{(q m+n p) k l}\right) \geq m l p k .
$$

We deduce

$$
\frac{v_{f g, N}\left(\alpha^{(q m+n p) k l}\right)}{(q m+n p) k l} \geq \frac{p m}{(q m+n p)}=\frac{1}{\frac{q}{p}+\frac{n}{m}},
$$

hence,

$$
\frac{1}{v_{f g, N}(\alpha)} \leq \frac{1}{\frac{p}{q}}+\frac{1}{\frac{m}{n}} .
$$

Taking the limit as $\frac{p}{q} \rightarrow \gamma$ and $\frac{m}{n} \rightarrow \delta$, we obtain

$$
\frac{1}{\bar{v}_{f g, N}(\alpha)} \leq \frac{1}{\bar{v}_{f, N}(\alpha)}+\frac{1}{\bar{v}_{g, N}(\alpha)} .
$$


(ii) It follows from inequality (a) that we have $\frac{1}{\bar{v}_{f^{n}, N}(\alpha)} \leq \frac{n}{\bar{v}_{f, N}(\alpha)}$, thus

$$
\bar{v}_{f^{n}, N}(\alpha) \geq \frac{1}{n} \bar{v}_{f, N}(\alpha)
$$

Suppose that $\bar{v}_{f^{n}, N}(\alpha)=\lambda$ and let $0<\frac{p}{q}<\lambda$. We can find an integer $n_{0}>0$ such that

$$
\frac{v_{f^{n}, N}\left(\alpha^{m}\right)}{m} \geq \frac{p}{q}
$$

for all $m \geq n_{0}$, hence $v_{f^{n}, N}\left(\alpha^{q m}\right) \geq m p$. We deduce $\alpha^{q m}(M) \subseteq I_{n m p} N$, therefore $v_{f, N}\left(\alpha^{q m}\right) \geq n m p$. We have

$$
\frac{v_{f, N}\left(\alpha^{q m}\right)}{q m} \geq n \frac{p}{q}
$$

hence,

$$
\bar{v}_{f, N}(\alpha) \geq n \frac{p}{q}
$$

Taking the limit as $\frac{p}{q} \rightarrow \lambda$, we get $\bar{v}_{f, N}(\alpha) \geq n \bar{v}_{f^{n}, N}(\alpha)$, thus

$$
\frac{1}{n} \bar{v}_{f, N}(\alpha) \geq \bar{v}_{f^{n}, N}(\alpha)
$$

Note that by a similar proof we obtain

$$
\frac{1}{n} \bar{v}_{f, N}(\alpha) \geq \bar{v}_{f}^{(n), N}(\alpha)
$$

It follows from Proposition 3.1 that we have $\bar{v}_{f^{n}, N} \leq \bar{v}_{f^{(n)}, N}$, since $f^{n} \leq f^{(n)}$. By inequalities $(*),(* *)$, and $(* * *)$, we obtain $\bar{v}_{f^{n}, N}(\alpha)=$ $\bar{v}_{f}^{(n), N}(\alpha)=\frac{1}{n} \bar{v}_{f, N}(\alpha)$. 
Corollary 3.1. Let $f=\left(I_{n}\right)$ be a filtration on the ring $A$ and $N$ be a sub-module of $M$ such that $\alpha(N) \subseteq N$ for all $\alpha \in \Lambda$. If $\bar{v}_{f, N}(\alpha) \neq+\infty$, then $\lim _{n \rightarrow+\infty} \bar{v}_{I_{n}, N}(\alpha)=0$ and $\bar{v}_{J, N}(\alpha)=0$ with $J=\bigcap_{m \geq 0} I_{m}$.

Proof. Let $n \in \mathbb{N}^{*}$ and $J=\bigcap_{m \geq 0} I_{m}$. Then $f_{J} \leq f_{I_{n}} \leq f^{(n)}$, since $J^{k} \subseteq I_{n}^{k} \subseteq I_{n k}$ for all $k \in \mathbb{N}$. By Proposition 3.1 (ii), we have $0 \leq \bar{v}_{f_{J}, N}$ $(\alpha) \leq \bar{v}_{I_{I_{n}}, N}(\alpha) \leq \bar{v}_{f}^{(n), N},(\alpha)$, hence $0 \leq \bar{v}_{J, N}(\alpha) \leq \bar{v}_{I_{n}, N}(\alpha) \leq \frac{1}{n} \bar{v}_{f, N}(\alpha)$. The last inequality follows from Theorem 3.1 (b). Taking the limit as $n \rightarrow+\infty$ we get $\bar{v}_{J, N}(\alpha)=\lim _{n \rightarrow+\infty} \bar{v}_{I_{n}, N}(\alpha)=0$.

Proposition 3.2. Let $f=\left(I_{n}\right), g=\left(J_{n}\right)$ be filtrations on the ring $A$ and $N$ be a sub-module of $M$ such that $\alpha(N) \subseteq N$ for all $\alpha \in \Lambda$.

(a) If $\bar{v}_{g, N}(\alpha) \neq 0$, then $\lim _{n \rightarrow+\infty} n \bar{v}_{f^{n} g, N}(\alpha)$ exists in $\overline{\mathbb{R}}$ and we have

$$
\lim _{n \rightarrow+\infty} n \bar{v}_{f^{n} g, N}(\alpha)=\bar{v}_{f, N}(\alpha)
$$

(b) If $\bar{v}_{g, N}(\alpha)=0$, then $\lim _{n \rightarrow+\infty} n \bar{v}_{f^{n} g, N}(\alpha)=0$.

(c) If $\sqrt{f N} \subseteq \sqrt{g N}$, then $\lim _{n \rightarrow+\infty} n \bar{v}_{f^{n} g, N}(\alpha)=\bar{v}_{f, N}(\alpha)$.

Proof. (a) Suppose that $\bar{v}_{g, N}(\alpha) \neq 0$.

By Proposition 3.1 (ii), we have $\bar{v}_{f^{n} g, N}(\alpha) \leq \bar{v}_{f^{n}, N}(\alpha)$ since $f^{n} g \leq f^{n}$. It follows that $n \bar{v}_{f^{n} g, N}(\alpha) \leq n \bar{v}_{f^{n}, N}(\alpha)=\bar{v}_{f, N}(\alpha)$, hence

$$
\lim \sup n \bar{v}_{f^{n} g, N}(\alpha) \leq \bar{v}_{f, N}(\alpha) .
$$

By Theorem 3.1, we have

$$
\frac{1}{n \bar{v}_{f^{n} g, N}(\alpha)} \leq \frac{1}{\bar{v}_{f, N}(\alpha)}+\frac{1}{n \bar{v}_{g, N}(\alpha)},
$$


thus

$$
\lim \sup \frac{1}{n \bar{v}_{f^{n} g, N}(\alpha)} \leq \lim \sup \left(\frac{1}{\bar{v}_{f, N}(\alpha)}+\frac{1}{n \bar{v}_{g, N}(\alpha)}\right)
$$

Therefore,

$$
\lim \sup \frac{1}{n \bar{v}_{f^{n} g, N}(\alpha)} \leq \frac{1}{\bar{v}_{f, N}(\alpha)}
$$

Since

$$
\lim \sup \frac{1}{n \bar{v}_{f^{n} g, N}(\alpha)}=\frac{1}{\lim \inf n \bar{v}_{f^{n} g, N}(\alpha)}
$$

we get

$$
\bar{v}_{f, N}(\alpha) \leq \liminf n \bar{v}_{f^{n} g, N}(\alpha)
$$

It follows from inequalities $(*)$ and $(* *)$ that $\lim _{n \rightarrow+\infty} n \bar{v}_{f^{n} g, N}(\alpha)$ exists and we have

$$
\lim _{n \rightarrow+\infty} n \bar{v}_{f^{n} g, N}(\alpha)=\bar{v}_{f, N}(\alpha)
$$

(b) Suppose that $\bar{v}_{g, N}(\alpha)=0$. We have $f^{n} g \leq g$ for all $n \geq 0$, hence $0 \leq \bar{v}_{f^{n} g, N} \leq \bar{v}_{g, N}$ by Proposition 3.1 (ii), therefore

$$
\lim _{n \rightarrow+\infty} n \bar{v}_{f^{n} g, N}(\alpha)=0 .
$$

(c) Let's assume that $\sqrt{f N} \subseteq \sqrt{g N}$. If $\alpha \notin \sqrt{g N}$, then $\bar{v}_{g, N}(\alpha)=\bar{v}_{f, N}(\alpha)=0$, so by (b) we have $\lim _{n \rightarrow+\infty} n \bar{v}_{f^{n} g, N}(\alpha)=\bar{v}_{f, N}(\alpha)$. If $\alpha \in \sqrt{g N}$, then $\bar{v}_{g, N}(\alpha) \neq 0$, so by (a) we have $\lim _{n \rightarrow+\infty} n \bar{v}_{f^{n} g, N}(\alpha)=\bar{v}_{f, N}(\alpha)$. 


\section{The f-Cloture of a Sub-Module}

Let $f=\left(I_{n}\right)_{n \in \mathbb{N}}, N, M$ and $\Lambda$ be as above. Let's put

$$
\Lambda_{n, f}=\left\{\alpha \in \Lambda / \bar{v}_{f, N}(\alpha) \geq n\right\} \quad \text { and } \quad \tilde{N}_{n}=\sum_{\bar{v}_{f, N}(\alpha) \geq n} \alpha(M)
$$

Remark 4.1. (1) We easily check that $\Lambda_{f}=\left(\Lambda_{n, f}\right)_{n \in \mathbb{N}}$ is a filtration on the ring $\Lambda$ and $\tilde{N}=\left(\tilde{N}_{n}\right)_{n \in \mathbb{N}}$ is a filtration on the $A$-module $M$.

(2) $\alpha\left(\tilde{N}_{n}\right) \subset \tilde{N}_{n}$ for all $\alpha \in \Lambda, n \in \mathbb{N}$.

(3) We have $\bar{v}_{f, N}(\alpha)=\bar{v}_{\Lambda_{f}}(\alpha)$ for all $\alpha \in \Lambda$ (we refer to [2] for details on Samuel numbers of filtrations on a ring). Indeed, (2) follows easily from the fact that $\alpha(N) \subset N$ for all $\alpha \in \Lambda$.

(3) Suppose $\bar{v}_{\Lambda_{f}}(\alpha) \neq+\infty$. Let us put $q_{n}=v_{\Lambda_{f}}\left(\alpha^{n}\right)$ for all $n \in \mathbb{N}$. We have $\alpha^{n} \in \Lambda_{q_{n}, f}$ and $\alpha^{n} \notin \Lambda_{q_{n}+1, f}$. It follows that

$$
q_{n} \leq \bar{v}_{f}, N\left(\alpha^{n}\right)<q_{n}+1 \text {. }
$$

For all $n \in \mathbb{N}$, therefore

$$
\frac{q_{n}}{n} \leq \bar{v}_{f, N}(\alpha)<\frac{q_{n}+1}{n},
$$

$n \in \mathbb{N}^{*}$. Taking the limit as $n \rightarrow+\infty$ we get $\bar{v}_{f, N}(\alpha)=\bar{v}_{\Lambda_{f}}(\alpha)$.

We easily check that if $\bar{v}_{\Lambda_{f}}(\alpha)=+\infty$, then $\bar{v}_{f, N}(\alpha)=+\infty$.

Definition 4.1. We put

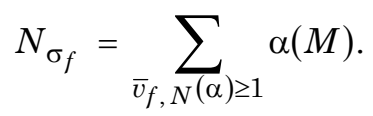

We call $N$ an $f$-normal submodule of $M$ if $N \subseteq N_{\sigma_{f}}$.

We have $N_{\sigma_{f}}=\tilde{N}_{1}$ and it is obvious that $N \subseteq \tilde{N}_{n} \Leftrightarrow N$ is $f^{n}$-normal. 
Theorem 4.1. Suppose that $A$ is a Noetherian ring and $M$ is finitely generated. If $N$ is $f^{n}$-normal, then $\bar{v}_{I_{n}, N}=\bar{v}_{I_{n}, \tilde{N}_{n}}$.

Proof. Since $N \subset \tilde{N}_{n}$, the inequality $\bar{v}_{I_{n}, N}(\alpha) \leq \bar{v}_{I_{n}, \tilde{N}_{n}}(\alpha)$ follows from Proposition 3.1 (i). Suppose $\lambda=\bar{v}_{I_{n}, \tilde{N}_{n}}(\alpha)>0$. Let $p, q \in \mathbb{N}^{*}$ such that $0<\frac{p}{q}<\bar{v}_{I_{n}, \widetilde{N}_{n}}(\alpha)$. Then $p<\bar{v}_{I_{n}, \widetilde{N}_{n}}\left(\alpha^{q}\right)$. We have $m p<v_{I_{n}, \widetilde{N}_{n}}\left(\alpha^{m q}\right)$ for $m$ large enough, hence

$$
\alpha^{m q}(M) \subseteq I_{n}^{m p} \tilde{N}_{n}=I_{n}^{m p} \sum_{\bar{v}_{f, N}(\alpha) \geq n} \alpha(M)
$$

for $m$ large enough. Since $M$ is finitely generated, there exists finitely many $\beta_{i}$, say $\beta_{1}, \ldots, \beta_{r}$ with $\bar{v}_{f, N}\left(\beta_{i}\right) \geq n$ and

$$
\alpha^{m q}(M) \subseteq I_{n}^{m p} \sum_{i=1}^{r} \beta_{i}(M)
$$

Let $u \in \mathbb{N}^{*}$. We have $\frac{u n}{u+1}<\bar{v}_{f, N}\left(\beta_{i}\right)$. Then $u n<\bar{v}_{f, N}\left(\beta_{i}^{u+1}\right)$. It follows that $k_{i} u n<v_{f, N}\left(\beta_{i}^{k_{i}(u+1)}\right)$ for $k_{i}$ large enough. Therefore,

$$
\beta_{i}^{k_{i}(u+1)}(M) \subset I_{k_{i} n u} N \subset N .
$$

Let's put $s_{i}=k_{i}(u+1)$ and $s=\sum s_{i}$. Then $\beta_{i}^{s_{i}}(M) \subset N$ for any $s_{i}$ and by $(*)$, we have

$$
\begin{gathered}
\alpha^{m q s}(M) \subseteq \alpha^{m q(s-1)}\left(I_{n}^{m p} \sum_{i}^{r} \beta_{i}(M)\right) \subseteq I_{n}^{m p} \alpha^{m q(s-2)}\left(\sum_{i}^{r} \beta_{i} \alpha^{m q}(M)\right) \\
\subseteq I_{n}^{2 m p} \alpha^{m q(s-2)}\left(\sum_{i, j}^{r} \beta_{i} \beta_{j}(M)\right) \subseteq \ldots \subseteq I_{n}^{s m p} \sum_{\sum_{i} t_{i}=s} \beta_{1}^{t_{1}} \ldots \beta_{r}^{t_{r}}(M) \subseteq I_{n}^{s m p} N .
\end{gathered}
$$


The last containment follows from the fact that at least one $t_{j} \geq s_{i}$. Thus

$$
\alpha^{m q s}(M) \subseteq I_{n}^{s m p} N
$$

It follows that $\frac{p}{q} \leq \bar{v}_{I_{n}, N}(\alpha)$. Taking the limit as $\frac{p}{q} \rightarrow \lambda$ we get $\bar{v}_{I_{n}, N}(\alpha) \geq \bar{v}_{I_{n}, \tilde{N}_{n}}(\alpha)$, hence $\bar{v}_{I_{n}, N}(\alpha)=\bar{v}_{I_{n}, \tilde{N}_{n}}(\alpha)$.

Corollary 4.1 ([5], Proposition 3.2). Let $M$ be a finitely generated A-module and $N$ be a submodule of $M$ such that $\alpha(N) \subseteq N$ for all $\alpha \in \Lambda$. Let $I$ be an ideal of the ring $A$ such that $N$ is I-normal (i.e., $f_{I}$-normal). Then

$$
\bar{v}_{I, N}=\bar{v}_{I, N_{\sigma_{I}}},
$$

with $N_{\sigma_{I}}=\sum_{\bar{v}_{I, N}(\alpha) \geq 1} \alpha(M)$.

Proof. By Theorem 4.1 (i), if $f=f_{I}=\left(I^{n}\right)_{n \in \mathbb{N}}$ and if $N$ is $I$-normal, then $\bar{v}_{I^{n}, N}=\bar{v}_{I^{n}, \tilde{N}_{n}}$. Taking $n=1$, we get $\bar{v}_{I, N}=\bar{v}_{I, N_{\sigma_{I}}}$, since $I^{1}=I$ and $\tilde{N}_{1}=N_{\sigma_{I}}$.

Let $A$ be a ring and $f=\left(I_{n}\right)_{\mathbb{N}}$ a filtration on $A$. Let $x \in A$. We put $v_{f}(x)=\sup \left\{n \in \mathbb{N} / x \in I_{n}\right\}$. It is known that $v_{f}: A \rightarrow \overline{\mathbb{R}}_{+}$is a pseudo valuation on $A$ and $\bar{v}_{f}(x)=\lim _{n \rightarrow+\infty} \frac{v_{f}\left(x^{n}\right)}{n}$ exists in $\overline{\mathbb{R}}_{+}$. We put $\bar{I}_{n}=\left\{x \in A / \bar{v}_{f}(x) \geq n\right\}$. Then $\bar{f}=\left(\bar{I}_{n}\right)_{n \in \mathbb{N}}$ is a filtration on $A$ and $f \leq \bar{f}$.

Theorem 4.2. Let $A$ be a Noetherian ring and $f=\left(I_{n}\right)_{n \in \mathbb{N}}, N, M$ and $\Lambda$ be as above. Suppose that $M$ is finitely generated and $N$ is f-normal. Then

$$
\bar{v}_{f, N}=\bar{v}_{f, N_{\sigma_{f}}} .
$$


Proof. Since $N \subseteq N_{\sigma_{f}}$ the inequality $\bar{v}_{f, N} \leq \bar{v}_{f, N_{\sigma_{f}}}$ follows from Proposition 3.1 (i). Let $\bar{v}_{f, N_{\sigma_{f}}}(\alpha)=\lambda>0$ and $0<\frac{p}{q}<\lambda$. We obtain $m p<v_{f, N_{\sigma_{f}}}\left(\alpha^{m q}\right)$ for $m$ large enough, hence

$$
\alpha^{m q}(M) \subset I_{m p} N_{\sigma_{f}}=I_{m p} \sum_{\bar{v}_{f}, N(\beta) \geq 1} \beta(M)
$$

for $m$ large enough. Since $A$ is Noetherian and $M$ is finitely generated, there exists finitely many $\beta_{i}$, say $\beta_{1}, \ldots, \beta_{r}$ with $\bar{v}_{f, N}\left(\beta_{i}\right) \geq 1$ and

$$
\alpha^{m q}(M) \subseteq I_{m p} \sum_{i=1}^{r} \beta_{i}(M)
$$

Since $1 \leq \bar{v}_{f, N}\left(\beta_{i}\right)$, for all $n \geq 1$, we have $\frac{n}{n+1}<\bar{v}_{f, N}\left(\beta_{i}\right)$. Then $n<\bar{v}_{f, N}\left(\beta_{i}^{n+1}\right)$. It follows that $k_{i} n<v_{f, N}\left(\beta_{i}^{k_{i}(n+1)}\right)$ for $k_{i}$ large enough. Therefore,

$$
\beta_{i}^{k_{i}(n+1)}(M) \subseteq I_{k_{i} n} N \subseteq N
$$

Let's put $s_{i}=k_{i}(n+1)$ and $s=\sum_{i=1}^{r} s_{i}$. Then $\beta_{i}^{s_{i}}(M) \subset N$ for any $s_{i}$ and by $(*)$, we have

$$
\begin{gathered}
\alpha^{m q s}(M) \subseteq \alpha^{m q(s-1)}\left(I_{m p} \sum_{i=1}^{r} \beta_{i}(M)\right) \subseteq I_{m p} \alpha^{m q(s-2)}\left(\sum_{i=1}^{r} \beta_{i} \alpha^{m q}(M)\right) \\
\subseteq I_{2 m p} \alpha^{m q(s-2)}\left(\sum_{1 \leq i, j \leq r} \beta_{i} \beta_{j}(M)\right) \subseteq \ldots \subseteq I_{s m p} \sum_{\sum_{i} t_{i}=s} \beta_{1}^{t_{1}} \ldots \beta_{r}^{t_{r}}(M) \subseteq I_{s m p} N .
\end{gathered}
$$

The last containment follows from the fact that at least one $t_{j} \geq s_{i}$. Thus

$$
\alpha^{m q s}(M) \subseteq I_{s m p} N
$$


It follows that $\frac{p}{q} \leq \bar{v}_{f, N}(\alpha)$. Taking the limit as $\frac{p}{q} \rightarrow \bar{v}_{f, N_{\sigma_{f}}}(\alpha)$ we get $\bar{v}_{f, N}(\alpha) \geq \bar{v}_{f, N_{\sigma_{f}}}(\alpha)$, hence $\bar{v}_{f, N}(\alpha)=\bar{v}_{f, N_{\sigma_{f}}}(\alpha)$.

Corollary 4.2. Let $A$ be a Noetherian ring and $M$ be a finitely generated A-module. Suppose that $f=\left(I_{n}\right)_{n \in \mathbb{N}}$ is a filtration on $A$ and that $N$ and $L$ are $\Lambda$-invariant and f-normal submodules of $M$. Then $N_{\sigma_{f}} \subseteq L_{\sigma_{f}}$ if and only if $\bar{v}_{f, N} \leq \bar{v}_{f, L}$.

Proof. It follows from Theorem 4.2 and Proposition 3.1 (i) that $\bar{v}_{f, N} \leq \bar{v}_{f, L}$ while the converse directly follows from the definition of -cloture.

Remark 4.2. Let $I$ be an ideal of the ring $A$ such that $N$ is $I$-normal (i.e., $f_{I}$-normal). By taking $f=f_{I}$ in Theorem 4.2, we have Corollary 4.1, page 17. By taking $f=f_{I}$ in Corollary 4.2, we have Proposition 3.5 in [5].

Let $A, f=\left(I_{n}\right)_{n \in \mathbb{N}}$ and $N, M, \Gamma$ be as above. Let $\alpha \in \Gamma$. Then $\left(\alpha^{n}(M)\right)_{n \in \mathbb{N}}$ is a filtration of submodules on $M$. We put $v_{f, \tilde{N}}(\alpha)=\left\{\begin{array}{l}\sup \left\{k \in \mathbb{N}^{*} / \alpha(M) \subseteq I_{k} \tilde{N}_{k}\right\} \text { if }\left\{k \in \mathbb{N}^{*} / \alpha(M) \subseteq I_{k} \tilde{N}_{k}\right\} \neq \emptyset \\ 0 \text { if }\left\{k \in \mathbb{N}^{*} / \alpha(M) \subseteq I_{k} \tilde{N}_{k}\right\}=\emptyset\end{array}\right.$

It is easily shown, as in [4]; Lemma 0.2.11, that $\bar{v}_{f \widetilde{N}}(\alpha)=\lim _{n \rightarrow+\infty}$ $\frac{v_{f} \widetilde{N}^{\left(\alpha^{n}\right)}}{n}$ exists in $\overline{\mathbb{R}}_{+}$.

Theorem 4.3. Let $A$ be a Noetherian ring and $M$ be a finitely generated A-module. If $N$ is $\Lambda$-invariant and $f^{n}$-normal for all $n \in \mathbb{N}$ then $\bar{v}_{f, N}(\alpha)=\bar{v}_{f \widetilde{N}}(\alpha)$. 
Proof. Since $N$ is $f^{n}$-normal, by $I_{n} N \subseteq I_{n} \tilde{N}_{n}$, we easily check that $\bar{v}_{f, N}(\alpha) \leq \bar{v}_{f \widetilde{N}}(\alpha)$. Suppose $\bar{v}_{f \widetilde{N}}(\alpha)>0$. Let $p, q \in \mathbb{N}^{*}$ such that $\frac{p}{q}<\bar{v}_{f \widetilde{N}}(\alpha)$. Then $p<\bar{v}_{f \widetilde{N}}\left(\alpha^{q}\right)$. We have $m p<v_{f \widetilde{N}}\left(\alpha^{m q}\right)$ for $m$ large enough, hence

$$
\alpha^{m q}(M) \subset I_{m p} \tilde{N}_{m p}=I_{m p} \sum_{\bar{v}_{f, N} \geq m p} \beta(M)
$$

for $m$ large enough. Since $A$ is Noetherian and $M$ is finitely generated, there exists finitely many $\beta_{i}$, say $\beta_{1}, \ldots, \beta_{r}$ with $\bar{v}_{f, N}\left(\beta_{i}\right) \geq m p$ and

$$
\alpha^{m q}(M) \subseteq I_{m p} \sum_{i=1}^{r} \beta_{i}(M)
$$

Since $m p \leq \bar{v}_{f, N}\left(\beta_{i}\right)$, for all $n \geq 1$, we have $\frac{n m p}{n+1}<\bar{v}_{f, N}\left(\beta_{i}\right)$. Then $n m p<\bar{v}_{f, N}\left(\beta_{i}^{n+1}\right)$. It follows that $k_{i} n m p<v_{f, N}\left(\beta_{i}^{k_{i}(n+1)}\right)$ for $k_{i}$ large enough. Therefore,

$$
\beta_{i}^{k_{i}(n+1)}(M) \subseteq I_{k_{i} n m p} N \subseteq N
$$

Let's put $s_{i}=k_{i}(n+1)$ and $s=\sum_{i}^{r} s_{i}$. Then $\beta_{i}^{s_{i}}(M) \subset N$ for any $s_{i}$ and by $(*)$, we have

$$
\begin{gathered}
\alpha^{m q s}(M) \subseteq \alpha^{m q(s-1)}\left(I_{m p} \sum_{i=1}^{r} \beta_{i}(M)\right) \subseteq I_{m p} \alpha^{m q(s-2)}\left(\sum_{i=1}^{r} \beta_{i} \alpha^{m q}(M)\right) \\
\subseteq I_{2 m p} \alpha^{m q(s-2)}\left(\sum_{1 \leq i, j \leq r} \beta_{i} \beta_{j}(M)\right) \subseteq \ldots \subseteq I_{s m p} \sum_{\sum_{i} t_{i}=s} \beta_{1}^{t_{1}} \ldots \beta_{r}^{t_{r}}(M) \subseteq I_{s m p} N .
\end{gathered}
$$

The last containment follows from the fact that at least one $t_{j} \geq s_{i}$. Thus

$$
\alpha^{m q s}(M) \subseteq I_{s m p} N
$$


It follows that $\frac{p}{q} \leq \bar{v}_{f, N}(\alpha)$. Taking the limit as $\frac{p}{q} \rightarrow \bar{v}_{f} \tilde{N}^{(\alpha)}$ we get, $\bar{v}_{f, N}(\alpha) \geq \bar{v}_{f \widetilde{N}}(\alpha)$, hence $\bar{v}_{f, N}(\alpha)=\bar{v}_{f \widetilde{N}}(\alpha)$.

Definition 4.2 ([5], 3.7; [3], Definition 2.3). Let $\Gamma$ be a set of submodules of an $A$-module $M$. We consider the following properties of a $\operatorname{map} \Gamma \rightarrow \Gamma ; N \mapsto N_{x}$ :

(a) $N \subseteq N_{x}$ for all $N \in \Gamma$;

(b) if $N \subseteq L$, then $N_{x} \subset L_{x}$;

(c) $\left(N_{x}\right)_{x}=N_{x}$;

(d) $I N \subseteq(I N)_{x}$ with $I$ an ideal of $A$.

Then $N \mapsto N_{x}$ is a closed operation on $\Gamma$ if (a)-(c) hold for all $N, L \in \Gamma$; it is a semiprime operation if (a)-(d) hold for all $N, L \in \Gamma$ and $I$ an ideal of $A$.

Proposition 4.1. Let $A$ be a Noetherian ring and $f=\left(I_{n}\right)_{n \in \mathbb{N}} a$ AP-filtration on $A$. Assume that $M$ is a finitely generated A-module. If $N$ and $L$ are $\Lambda$-invariant and f-normal submodules of $M$, then we have:

(i) if $N \subseteq L$, then $N_{\sigma_{f}} \subseteq L_{\sigma_{f}}$;

(ii) $\left(N_{\sigma_{f}}\right)_{\sigma_{f}}=N_{\sigma_{f}}$;

(iii) $I_{n} N_{\sigma_{f}} \subseteq\left(I_{n} N\right)_{\sigma_{f}}$ for all $n \in \mathbb{N}^{*}$.

Proof. (i) Follows directly from Corollary 4.2 and Proposition 3.1.

(ii) Follows from Theorem 4.2.

(iii) By Proposition 3.1 (iii), we have $\bar{v}_{f, N}=\bar{v}_{f, I_{n} N}$, it follows from Corollary 4.2 that $N_{\sigma_{f}}=\left(I_{n} N\right)_{\sigma_{f}}$, therefore $I_{n} N_{\sigma_{f}} \subseteq N_{\sigma_{f}}=\left(I_{n} N\right)_{\sigma_{f}}$, hence $I_{n} N_{\sigma_{f}} \subseteq\left(I_{n} N\right)_{\sigma_{f}}$. 
Suppose that $A$ is a Noetherian ring and $f=\left(I_{n}\right)_{n \in \mathbb{N}}$ is an AP-filtration on $A$. Let $\Gamma_{f, \Lambda}$ be the set of all $\Lambda$-invariant and $f$-normal submodules of $M$. Assume that $M$ is a finitely generated $A$-module.

Corollary 4.3. The map $\Gamma_{f, \Lambda} \rightarrow \Gamma_{f, \Lambda} ; N \mapsto N_{\sigma_{f}}$ is a semiprime operation on $\Gamma_{f, \Lambda}$.

Proof. The proof follows directly from the definition of $f$-normality and Proposition 4.1.

Remark 4.3. We refer to Notation 2.1. Let $I$ be an ideal of $A$. By taking $f=f_{I}$, we get Proposition 3.8 in [5].

Proposition 4.2. Let $A$ be a Noetherian ring and $J$ be an ideal of $A$. Assume that $M$ is a finitely generated A-module and $N$ and $L$ are $f=\left(I_{n}\right)$-normal submodules of $M$. If $\sqrt{f N} \subseteq \operatorname{Rad}\left(J N:_{\Lambda} M\right)$ and $J N$ is f-normal, then

$$
J N_{\sigma_{f}} \subseteq(J N)_{\sigma_{f}}
$$

Proof. We consider the filtration $g=\left(J_{n}\right)_{n \in \mathbb{N}}$ with $J_{n}=J$ for all $n \in \mathbb{N}^{*}$ and $J_{0}=A$. Then $f^{p} g=\left(I_{n}^{p} J_{n}\right)_{n \in \mathbb{N}}$. We easily check that $\bar{v}_{f^{p} g, N}(\alpha)=\bar{v}_{f^{p}, J N}(\alpha)$ for all $\alpha \in \Lambda$ and $p \in \mathbb{N}^{*}$. By Proposition 3.2, we have $\bar{v}_{f, N}(\alpha)=\lim _{p \rightarrow+\infty} p \bar{v}_{f}^{p g, N}(\alpha) \quad$ since $\quad \sqrt{f N} \subseteq \operatorname{Rad}\left(J N:_{\Lambda} M\right)$. Therefore $\bar{v}_{f, N}(\alpha)=\lim _{p \rightarrow+\infty} p \bar{v}_{f^{p}, J N}(\alpha)=\bar{v}_{f, J N}(\alpha)$, thus $\bar{v}_{f, N}(\alpha)=$ $\bar{v}_{f, J N}(\alpha)$. By Theorem 4.2, we obtain $\bar{v}_{f, N_{\sigma_{f}}}=\bar{v}_{f,(J N)_{\sigma_{f}}}$. It follows from Corollary 4.2 that $N_{\sigma_{f}}=(J N)_{\sigma_{f}}$, hence $J N_{\sigma_{f}} \subseteq N_{\sigma_{f}}=(J N)_{\sigma_{f}}$. 


\section{References}

[1] P. Ayegnon and Daouda Sangare, Generalized Samuel numbers and AP-filtrations, Journal of Pure and Applied Algebra 65 (1990), 1-13, North-Holland.

[2] P. Ayegnon and H. Dichi, Extensions aux filtrations des nombres de Samuel associés aux idéaux, Communication in Algebra 22(9) (1994), 3249-3263.

[3] David E. Rush, Asymptotic primes and integral closure in modules, Quart J. Math. Oxford Ser. (2) 43(4) (1992), 477-499.

[4] M. Lejeune and B. Teissier, Clôture intégrale des idéaux et équisingularité, Séminaire Centre de Mathématique Ecole Polytechnique, 1974.

[5] G. Naudé and C. Naudé, Asymptotic completion of a module with respect to certain ideal, Comm. Algebra 24(5) (1996), 1549-1564.

[6] J. W. Petro, Some Results in the Theory in Pseudo-Valuations, Ph. D. Dissertation, State University of Iowa, Iowa City, Iowa, 1961.

[7] P. Samuel, Some asymptotic properties of powers of ideals, Ann. of Math. 56 (1952), 11-21.

[8] S. Ouattara, E. D. Akeke and P. Ayegnon, Another generalised Samuel number $\hat{a}_{f}(g)$ on a semi-ring, JP Journal of Algebra, Number Theory and Applications 19(2) (2010), 185-201. 